\title{
Antibiotics Resistance Genes Screening and Comparative Genomics Analysis of Commensal Escherichia coli Isolated from Poultry Farms between China and Sudan
}

\author{
Sheikheldin A. Abdelgader, ${ }^{1,2}$ Donglin Shi, ${ }^{1}$ Mianmian Chen, ${ }^{1}$ Lei Zhang, \\ Hassan M. A. Hejair, ${ }^{1}$ Umair Muhammad, ${ }^{3}$ Huochun Yao, ${ }^{1}$ and Wei Zhang $\mathbb{D}^{1}$ \\ ${ }^{1}$ College of Veterinary Medicine, Nanjing Agricultural University, Nanjing 210095, Jiangsu Province, China \\ ${ }^{2}$ College of Animal Production, Bahri University, Khartoum, Sudan \\ ${ }^{3}$ College of Food Science and Technology, Nanjing Agricultural University, Nanjing 210095, Jiangsu Province, China
}

Correspondence should be addressed to Wei Zhang; vszw@njau.edu.cn

Received 5 May 2018; Revised 26 July 2018; Accepted 9 August 2018; Published 26 August 2018

Academic Editor: Stanley Brul

Copyright (C) 2018 Sheikheldin A. Abdelgader et al. This is an open access article distributed under the Creative Commons Attribution License, which permits unrestricted use, distribution, and reproduction in any medium, provided the original work is properly cited.

\begin{abstract}
Escherichia coli (E. coli) strains, from the gut of animals and humans, harbor wide range of drug resistance genes. A comparative study is conducted on the intestinal E. coli from fecal samples of healthy chicken from China and Sudan in order to monitor the antimicrobial sensitivity pattern. A number of $250 \mathrm{E}$. coli isolates from chicken farms, including 120 from China and 130 from Sudan, were isolated and identified. All isolates were subjected to susceptibility tests against 10 antibiotics and the distribution of antibiotic resistant genes was confirmed by PCR amplification, involving genes such as ampC, tet $A, p K D 13, \operatorname{acr} A, \operatorname{erm} A, \operatorname{erm} B, \operatorname{erm} C$, tetB, $m p h A$, aadA14, aadA1, aac3-1, and aac3- III. Many isolates were found to exhibit resistance against more than one antibiotic. However, the Chinese isolates showed more antibiotics resistance and resistance genes compared to the Sudanese isolates. For better understanding of the multidrug resistance factors, we conducted whole genome analyses of E. coli D107 isolated from China, which revealed that the genome possesses multiple resistance genes including tetracycline, erythromycin, and kanamycin. Furthermore, E. coli D4 isolate from Sudan was more sensitive to antibiotics such as erythromycin, tetracycline, and gentamicin. After analysis by RAST and MAUVE, the two strains showed $89 \%$ average nucleotide identity. However, the genomes mostly differed at the number of antibiotics-related genes, as the genome of D107 revealed a considerable number of antibiotics resistance genes such as ermA and $m p h D$ which were found to be absent in $\mathrm{D} 4$ genome. These outcomes provided confirmation that the poultry farms environment in different countries (China and Sudan) may serve as a potential reservoir of antimicrobial resistance genes and also indicated the evolutionary differences of strains in terms of resistant genes expression.
\end{abstract}

\section{Introduction}

Antibiotic resistance is one of the upcoming crucial concerns to global health care [1], and the spread of antibiotic-resistant bacteria stands as one of the most dangerous global health care issues to human health [2]. This resistance among bacteria is now recognized to have a considerable effect in rising morbidity, mortality, and costs associated with major public health problems [3]. Increasing antimicrobial resistance problems do not only affect developed countries but also affect nonindustrialized countries, where antibiotics resistance issues are more challenging, because of the lack of well-organized antimicrobial usage policies and the need for optimal hygiene situation and contagion control practices [4]. The use of antimicrobials in animal farm does not only induce the resistance in the pathogens but also produce resistance in the commensal bacterial of individuals or groups $[5,6]$. Description of antimicrobial resistance determinants in bacteria at the genetic level plays a critical role in understanding and possibly controlling the resistance [7]. Moreover, it is clear that genetic exchange system and the ability of $E$. coli to transfer and propagate genes between humans and animals may make it a significant vector for the spread of rapidly dispersed resistance genes $[8,9]$. 
TABLE 1: Class, concentration range, and resistant breakpoints of tested antibiotics.

\begin{tabular}{|c|c|c|}
\hline $\begin{array}{l}\text { Class or } \\
\text { antibiotics } \\
\end{array}$ & $\begin{array}{c}\text { Dilution range tested } \\
(\mu \mathrm{g} / \mathrm{mL})\end{array}$ & $\begin{array}{c}\text { Resistance } \\
\text { breakpoint }(\mu \mathrm{g} / \mathrm{mL}) \\
\end{array}$ \\
\hline Ampicillin & $0.25-32$ & $\geq 32$ \\
\hline Tetracycline & $1.0-32$ & $\geq 16$ \\
\hline Kanamycin & $0.25-64$ & $\geq 64$ \\
\hline Ciprofloxacin & $0.01-15$ & $\geq 04$ \\
\hline Erythromycin & $0.25-8.0$ & $\geq 08$ \\
\hline Doxycycline & $1.0-16$ & $\geq 16$ \\
\hline Cefotaxime & $0.01-5.0$ & $\geq 04$ \\
\hline Spectinomycin & $1.0-128$ & $\geq 128$ \\
\hline Gentamicin & $0.25-8.0$ & $\geq 08$ \\
\hline Streptomycin & $1.0-128$ & $\geq 128$ \\
\hline
\end{tabular}

Genomes resistant to antimicrobials might have emerged from continuous accumulation of multiple mutations [12]. Hence, whole-genome sequence (WGS) of microorganisms has become an important tool for antibiotics resistance screening and, thus, provides rapid identification of antibiotic resistance mechanisms. Moreover, WGS also enables evaluating the number of mutations and functions of the mutated genes $[13,14]$. Sequencing the entire genome is found to be helpful in many antimicrobial applications such as new antibiotics development, diagnostic tests, the management of presently available antibiotics, and clarifying the factors promoting the emergence and resistance of pathogenic bacteria $[15,16]$. During the last two decades, the use of antibiotics in poultry farming has changed, as the utilization of antibiotics as growth promoters is banned or severely restricted in some countries, which caused distinctive differences between countries in regard to prevalence of antibiotic resistance [17, 18].

The objective of our study was to comparatively determine the antibiotic resistance in commensal $E$. coli isolated from chicken's farms between China and Sudan. We utilized WGS and polymerase chain reaction (PCR) techniques to study antimicrobial resistance genes and highlighted the importance of identifying the antibiotics resistance changes in the natural microbiota due to the overuse of antimicrobials by commercial poultry meat producers.

\section{Materials and Methods}

2.1. Samples Collection. A total of 500 cloacal swab samples were collected from commercial chicken farms located in Khartoum, Sudan $(n=250)$, and Nanjing, China $(n=250)$, in June, 2016. All samples were swabbed with a sterile cotton swab (Xuzhou Kanger Company, Xuzhou, China). After collection, samples were stored in ice-cold sterile containers during transportation from the farm to the laboratory. The samples collected from Sudan were transferred in LB medium (Sigma Aldrich, China) to the College of Veterinary Medicine Nanjing Agriculture University, China, within two days after collection; for isolation and identification. All the samples collected from China and Sudan were handled at the same time.
2.2. Bacterial Isolation. Samples collected from both countries were inoculated ( $5 \mu \mathrm{L}$ inoculums) in LB medium and stored at $4^{\circ} \mathrm{C}$. Fecal coliform isolates were further characterized by streaking on MacConkey agar (Qingdao hope biotechnology co. ltd., China) and incubated overnight at $37^{\circ} \mathrm{C}$. The phylogenetic classification of the bacteria isolates was conducted by multiplex PCR and assigned to the 4 major $E$. coli bacteria groups A, B1, B2, and group D according to the $16 \mathrm{~S}$ rRNA analysis.

Based on the initial biochemical tests, a total of $250 \mathrm{E}$. coli isolates (120 samples from China and 130 samples from Sudan) were selected and stored in sterile containers for further tests.

2.3. Antibiotics Agents. All the bacterial isolates were tested for antibiotics resistance including ampicillin, cefotaxime, kanamycin, erythromycin, doxycycline, ciprofloxacin, tetracycline, gentamicin, streptomycin, and spectinomycin. All antimicrobials were purchased from Sigma-Aldrich, China. Antibiotics were prepared and diluted in the LB medium to concentrations ranging from $0.01 \mu \mathrm{g} / \mathrm{mL}$ to $128 \mu \mathrm{g} / \mathrm{mL}$ (Table 1). Isolates which showed resistance to two or more antimicrobial agents were defined as multidrug-resistant.

2.4. Antibiotics Testing. Antibiotic susceptibilities of the E. coli isolates were determined by minimum inhibitory concentration (MIC) test and were carried out by the agar dilution method according to Clinical and Laboratory Standards Institute (CLSI) recommendations [19].

The MIC was reported as the lowest test concentration extract that showed no visible bacterial growth. The plate wells with a complete inhibition of macroscopic growth were recorded as MIC against the tested strain. The density of the suspension to contain colony forming units (CFU)/mL was adjusted by comparison with a $0.5 \mathrm{McF}$ arland turbidity standard. Tested antibiotics, concentration ranges, and resistance MIC points are listed in Table 1, and E. coli ATCC 25922 was used as the control organism.

2.5. Detection of Antibiotics Resistance Genes. The PCR amplifications were carried out using PCR primers listed in Table 2, for the detection of 13 different antibiotics resistance 


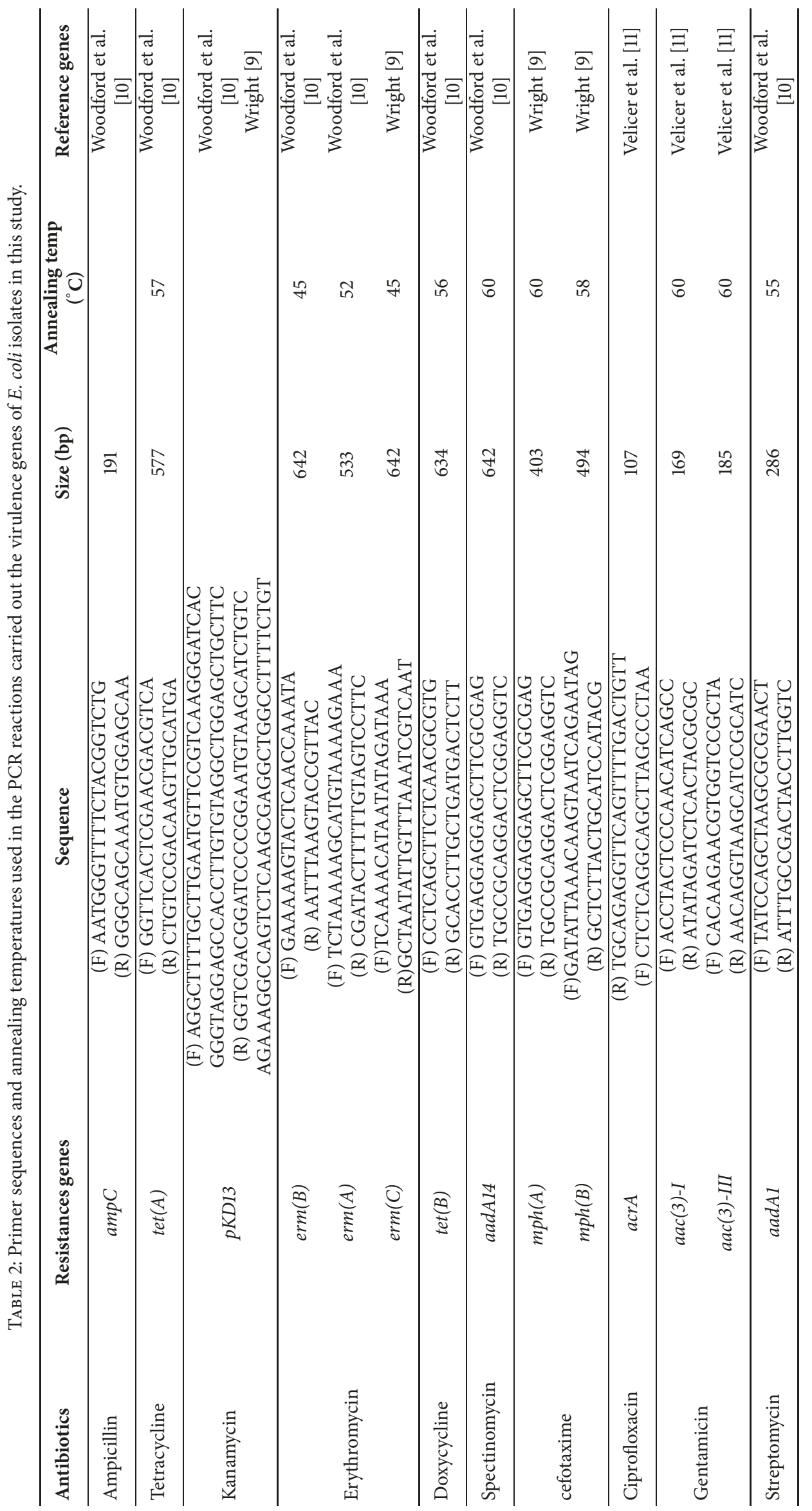


TABLE 3: Phenotypic pattern of commensal E. coli isolates from poultry farms in China and Sudan to 10 antibiotics agents, included in this study.

\begin{tabular}{|c|c|c|c|c|c|c|}
\hline \multirow{2}{*}{ Antibiotics } & \multicolumn{2}{|c|}{ Sensitive \% } & \multicolumn{2}{|c|}{ Intermediate \% } & \multicolumn{2}{|c|}{ Resistant \% } \\
\hline & China & Sudan & China & Sudan & China & Sudan \\
\hline Ampicillin & 10.0 & 33.3 & 15.0 & 37.5 & 75.2 & 29.2 \\
\hline Spectinomycin & 20.0 & 40.2 & 30.0 & 22.3 & 50.0 & 37.6 \\
\hline Kanamycin & 15.0 & 42.0 & 20.0 & 16.0 & 65.2 & 42.0 \\
\hline Erythromycin & 15.0 & 37.2 & 19.8 & 29.2 & 65.2 & 33.6 \\
\hline Cefotaxime & 15.0 & 33.0 & 39.0 & 33.3 & 46.0 & 33.6 \\
\hline Doxycycline & 29.8 & 45.8 & 25.0 & 29.0 & 45.2 & 25.2 \\
\hline Tetracycline & 5.0 & 29.1 & 15.0 & 16.5 & 80.0 & 54.4 \\
\hline Ciprofloxacin & 20.0 & 25.0 & 24.8 & 25.0 & 46.0 & 50.0 \\
\hline Gentamicin & 24.4 & 30.0 & 34.0 & 37.0 & 41.6 & 33.6 \\
\hline
\end{tabular}

genes $A R G s$, such as ampicillin ampC, tetracycline tetA, kanamycin $p K D 13$, ciprofloxacin acrA, erythromycin erm $A$, erm $B$, erm $C$, doxycycline tet $B$, cefotaxime $m p h A$, spectinomycin aadA14, streptomycin aadA1, and gentamicin aac3-1, aac3- III.

2.6. Genomic DNA Extraction. The total bacterial DNA was extracted by using commercial bacterial DNA extraction kit (Omega Co., China) according to the kit manufacturer's instructions. Briefly, $1.5 \mathrm{~mL}$ of $E$. coli isolate (grown overnight in LB medium) was pelleted in Eppendorf tube by microcentrifuge at $10,000 \times \mathrm{g}$ at $\max$ speed for $10 \mathrm{~min}$. The supernatant was discarded followed by addition of $100 \mu \mathrm{L}$ TE buffer and vortexed to completely resuspended the cell pellet. Lysozyme $(10 \mu \mathrm{L})$ was added and the solution was incubated at $57^{\circ} \mathrm{C}$ for 10 minutes. Then, $20 \mu \mathrm{L}$ of proteinase $\mathrm{K}$ solution was added, samples were vortexed, and the cell solution was incubated at $55^{\circ} \mathrm{C}$ shaking water bath for lysis $6 \mathrm{hrs}$. After the lysis, $5 \mu \mathrm{L}$ of the RNase was added and the tubes were incubated at room temperature for 5 minutes. This was followed by the addition of $220 \mu \mathrm{L}$ of BDL buffer; then, samples were vortexed and incubated at $65^{\circ} \mathrm{C}$ for 10 minutes. The genomic DNA was concentrated by the addition of $220 \mu \mathrm{L}$ of $100 \%$ ethanol, vortexed for 20 seconds, and centrifuged for $1 \mathrm{~min}$ at $10,000 \times \mathrm{g}$. Then, $500 \mu \mathrm{L}$ HBC buffer was added and centrifuged for $1 \mathrm{~min}$ at $10,000 \times \mathrm{g}$ for washing. The purified DNA was eluted in a fresh $1.5 \mathrm{~mL}$ microcentrifuge tube using $100 \mu \mathrm{L}$ elution buffer, 171 , and kept at room temperature for 5 minutes, and centrifuged for $1 \mathrm{~min}$ at $10,000 \times \mathrm{g}$. Finally, the DNA concentration was evaluated using nanodrop spectrophotometer before being stored at $-20^{\circ} \mathrm{C}$.

2.7. Genome Annotations and Comparison. DNA sequencing runs of $E$. coli isolates were completed for both genomes of D107 (resistant) strain from China and D4 (sensitive) strain from Sudan. Open reading frames annotation and comparative gene clusters analysis was conducted using the rapid annotation subsystem technology (RAST version 4.0). Gene sequence alignment was performed using Genedoc (version 2.6).

\section{Results and Discussion}

3.1. Antibiotics Resistance Testing. Antimicrobial resistance test of all $E$. coli isolates were determined by minimum inhibitory concentration (MIC). Classification and percentage distribution of antibiotic resistance in commensal $E$. coli isolates against 10 different antibiotics is presented in Table 3. All E. coli isolates were resistant to at least one antibiotic; these results are consistent with other previous studies on commensal E. coli, where commensal E. coli exhibited high prevalence of resistance to commonly used antibiotics in livestock [20, 21] (Miles et al., 2016). Furthermore, about $75.5 \%$ of bacterial isolate were resistant to more than two antibiotics (multidrug-resistant). Among examined individual antibiotics, tetracycline resistance was the highest percentage found in both isolates from China and Sudan at of $80 \%$ and $54.4 \%$, respectively. This high rate of resistance in commensal $E$. coli from poultry was also demonstrated in the study of Cizman [22]. Tetracycline is commonly used antibiotic against commensal E. coli from chicken farms in the above studied two countries. Another significantly high resistance was found towards ampicillin at $75.2 \%$ of isolates from China; however, the isolates from Sudan were significantly lower in percentage (29.2\%). This is followed by the resistance to ampicillin, kanamycin, and erythromycin. Moreover, about $62 \%$ of the E. coli isolates resistant to ampicillin also showed resistance against tetracycline.

The overall phenotypic resistance comparison reveals significant higher rates of antibiotic resistant $E$. coli isolates from China compared to the Sudan isolates except in the ciprofloxacin where the Sudanese isolate showed higher resistance, which might be due to the variation of antibiotics overuse between both countries.

3.2. Distribution of E. coli Isolates Resistance Genes. PCR amplifications were performed to detect 13 virulence genes using their appropriate primers. The distribution of identified virulence genes is presented in Figure 1. Tetracycline resistant gene tet $A$ was found to be highly distributed in isolates from 


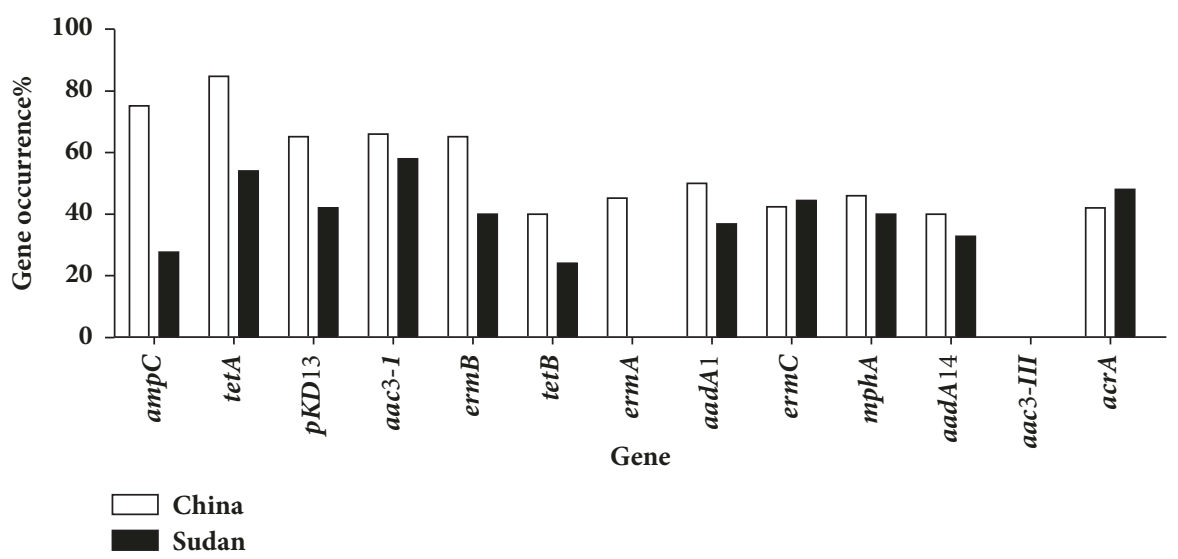

FIGURE 1: Distribution of resistance genes among commensal Escherichia coli isolates, from poultry farms (China and Sudan).

TABLE 4: Inventory of antibiotic resistance genes identified in whole E. coli genomes (D107 and D4).

\begin{tabular}{lcc}
\hline Antibiotics genes & D 107 & D 4 \\
\hline ampC & 1 & 1 \\
tetA & 1 & 1 \\
pKD13 & 1 & 1 \\
aac3-1 & 1 & 1 \\
ermA & 1 & 0 \\
qnrA1 & 0 & 0 \\
ermB & 1 & 1 \\
tetB & 1 & 1 \\
aadA1 & 1 & 1 \\
gyr & 1 & 1 \\
$m p h A$ & 1 & 1 \\
aadA14 & 1 & 0 \\
aac3- III & 1 & 1 \\
acrA & 0 & 0 \\
mphD & 1 & \\
\hline
\end{tabular}

China and Sudan at $84 \%$ and $54 \%$, respectively, which is comparable to some studies reporting higher frequencies of tet $A$ and tet $B[23,24]$. This percentage is followed by the ampicillin resistance gene $(a m p C)$ at $75 \%$ and $27.6 \%$, respectively. The detection of resistance genes was found to be in agreement with the phenotypic resistance data in Table 3, suggesting that these genes play significant role in the tolerance against the tested antibiotic. Moreover, about $72 \%$ of the tet $A$ resistance isolates were also resistant to $a m p C$, and around $35 \%$ of isolates possessing ampC/pKD13 genes in their genome were found to possess tet $A$ gene, indicating significantly high prevalence of these multidrug resistance isolates. The occurrence of remaining genes in the genome of tested isolates was present in a range from $34 \%$ to $66 \%$, except for erythromycin ermA in Sudan isolates and gentamicin (aac3- III) in both countries which were not detected, while the $p K D 13$ genes were identified in $65 \%$ and $42 \%$ of isolates from China and Sudan, respectively. Therefore, this indicates that local and the geographical localization factors can play a major role in the appearance of some antimicrobial resistance genes. It has been mentioned that sensitive strains harbor antibiotic(s) resistant gene(s) might express this resistant and generate strains that are likely to be resistant to those antibiotics [25].

3.3. Comparative Genomes Sequence. In order to determine the extent of DNA sequencing coverage, TBLASTN searches were performed against the whole genome sequences of D107 strain from China and D4 strain from Sudan. In general, among these two E. coli genomes, the chromosomes of Sudanese strain (more antibiotic sensitive) were found to have smaller genome size $(4,742,490 \mathrm{bp})$ and consisted of 4179 coding DNA sequences (CDS), whereas the Chinese strain has 5,111,357 bp and 5193 CDS. Figure 2 represents different gene groups categorized by RAST (RAST.nmpdr.org) annotation tool. The genome sequence of commensal $E$. coli strain D107 isolated from China revealed a wide array of antimicrobial resistance genes compared with the whole genome of the E. coli D4 strain.

The antimicrobial resistance genes identified in whole genomes sequence of E. coli D107 and D4 are listed in Table 4 . The comparative sequence analysis of both D107 and 

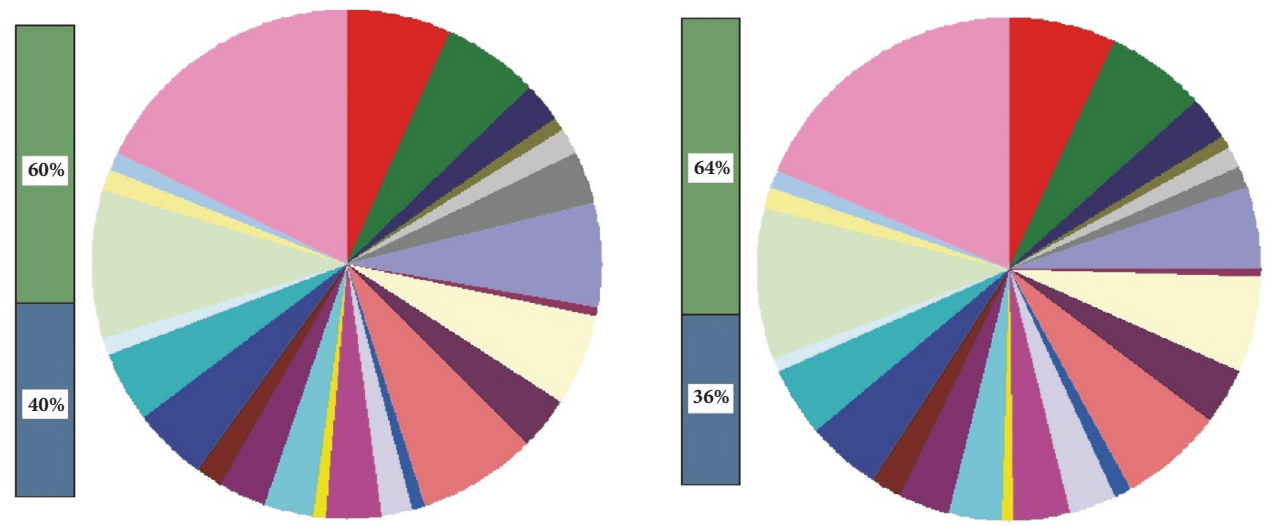

System Features count

Cofactors, Vitamins, Prosthetic Groups, Pigments (286)

Cell Wall and Capsule (254)

Virulence, Disease and Defense (109)

Potassium metabolism (31)

Photosynthesis (0)

Miscellaneous (65)

Phages, Prophages, Transposable elements, Plasmids (137)

Membrane Transport (278)

Iron acquisition and metabolism (23)

RNA Metabolism (248)

Nucleosides and Nucleotides (145)

Protein Metabolism (306)

Cell Division and Cell Cycle (39)

Motility and Chemotaxis (80)

Regulation and Cell signaling (154)

Secondary Metabolism (26)

DNA Metabolism (126)

Fatty Acids, Lipids, and Isoprenoids (129)

Nitrogen Metabolism (75)

Dormancy and Sporulation (5)

Respiration (199)

Stress Response (179)

Metabolism of Aromatic Compounds (45)

Amino Acids and Derivatives (394)

Sulfur Metabolism (58)

Phosphorus Metabolism (53)

Carbohydrates (729)

(a)

System Features count

Cofactors, Vitamins, Prosthetic Groups, Pigments (286)

Cell Wall and Capsule (265)

Virulence, Disease and Defense (108)

Potassium metabolism (29)

Photosynthesis (0)

Miscellaneous (65)

Phages, Prophages, Transposable elements, Plasmids (50)

Membrane Transport (220)

Iron acquisition and metabolism (22)

RNA Metabolism (248)

Nucleosides and Nucleotides (147)

Protein Metabolism (271)

Cell Division and Cell Cycle (38)

Motility and Chemotaxis (129)

Regulation and Cell signaling (148)

Secondary Metabolism (26)

DNA Metabolism (141)

Fatty Acids, Lipids, and Isoprenoids (132)

Nitrogen Metabolism (76)

Dormancy and Sporulation (5)

Respiration (192)

Stress Response (182)

Metabolism of Aromatic Compounds (30)

Amino Acids and Derivatives (394)

Sulfur Metabolism (56)

Phosphorus Metabolism (53)

Carbohydrates (741)

(b)

Figure 2: Genomic maps showing deferent group of genes functions categories, annotated by RAST. (a) E. coli D107 (China strain); (b) E. coli D4 (Sudan strain). The number of shared genes and the number of unique genes and genes shared between two strains are shown.

D4 genomes (Figure 3) revealed that erythromycin (ermA) and cefotaxime gene $(m p h D)$ antibiotics resistant genes were found in the D107 while being absent in the E. coli from the D4 isolate. The missing resistance genes from antimicrobial susceptible strain (D4) suggests that it has various plasmid complement that any antimicrobial resistance determinants were either missing or never obtained. However, quinolone (qnrA1) and gentamicin (aac3-III) resistance genes were not found in both genomes.

The detection of the differences between the two genomes holds a great potential for understanding the development of risky E. coli resistant strains from the Chinese isolate.
The chromosomal backbones of the two E. coli strains are different as in Figure 3. Although D107 and D4 (both are commensal bacteria) have highly various antimicrobial profiles of high and low susceptibility, respectively, this suggests that transferring exogenous or horizontal genes is a key mechanism for the acquisition of antimicrobial resistance. This is implication is reasonable, since many antimicrobial resistance genes determinants are transmitted by moving plasmids or mobile elements, particularly for gut microorganisms [26].

The specific gene sequence comparison in between D107 and D4 strains is exemplified for the ampC gene (Figure 4). 


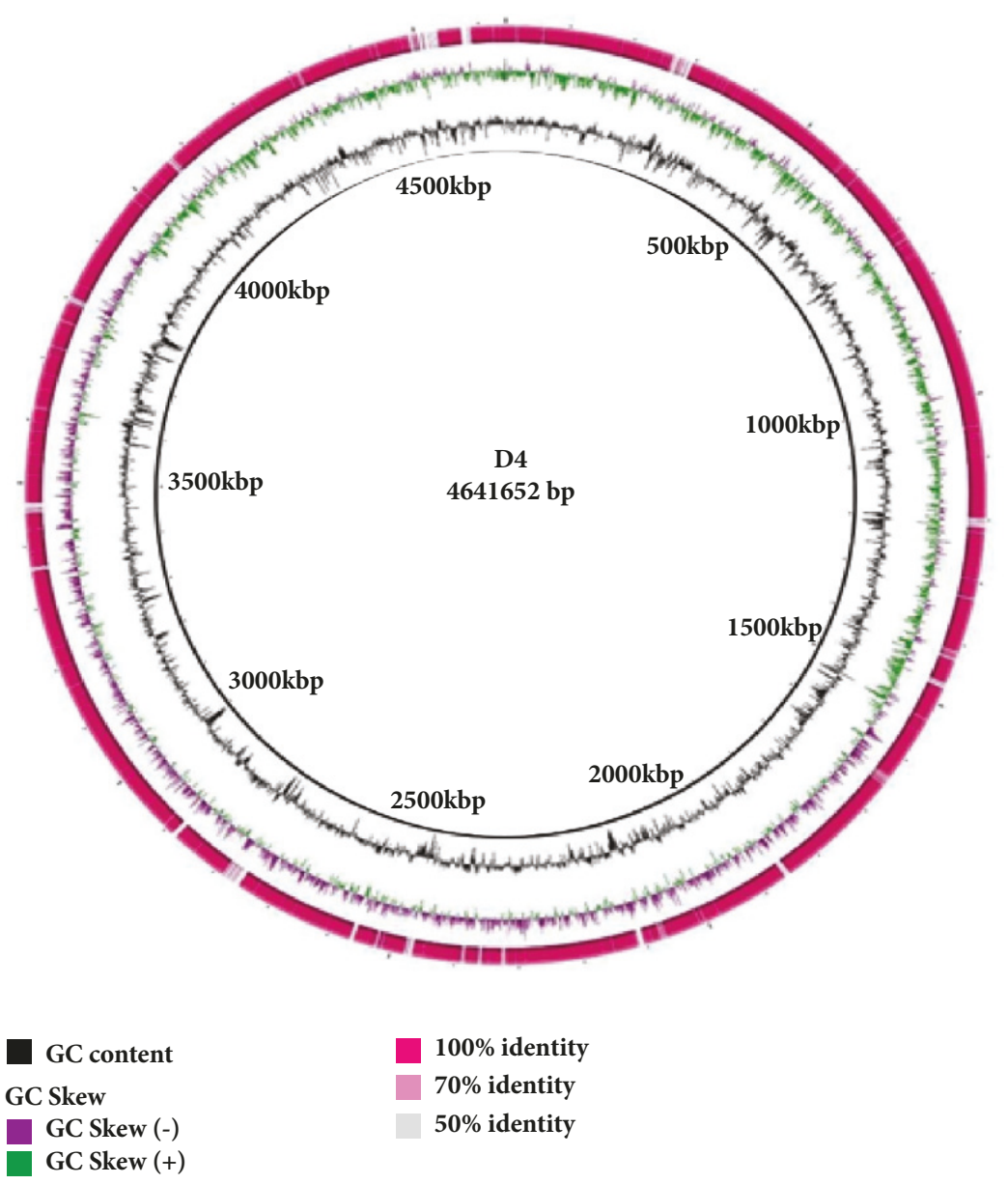

Figure 3: Circular map of D107 and D4 Genomic comparison. The outer circle shows identity position of both genomes. The second circle shows the D107 genome and inner circle shows D4 genome. Gaps seen in D4 indicate that sequence is missing in this isolate but present in D107.

Although the gene was present in the genome of both strains, the phenotypic resistance varied significantly which could indicate a strong engagement between antibiotics resistance genes and an elevated mutation rate. Moreover, many studies have observed that the acquisition of a mutation provides antibiotic resistance genes [26].

\section{Conclusion}

Herein we report a comparative study on the antibiotic resistance isolates of commensal E. coli, evaluating genes associated with antibiotic resistance from Chinese and Sudanese poultry farms. Clear variation in phenotypic resistance patterns was found, as isolates from China were significantly more tolerant to a wide array of antibiotics, which is supported by the detection of specific genes and genomewide comparative analysis. The results indicate the variations of irrational utilization of these antimicrobial agents in animal farms for treatments or as growing promoters. We are currently exploring the interplay between the genetic makeup of resistant strains and their expressed phenotypic resistance.

\section{Data Availability}

The data used to support the findings of this study are available from the corresponding author upon request.

\section{Conflicts of Interest}

The authors declare that they have no conflicts of interest.

\section{Acknowledgments}

The authors thank Dr. Faisal Nureldin Awad (National Food Research Centre, Sudan) for the valuable comments and language editing of this manuscript. This work was supported by grants from the National Natural Science Foundation of China (no. 31322054) and the Priority Academic Program Development of Jiangsu Higher Education Institutions. 
$\begin{array}{cl}\text { D107 : } & \text { TTACTGTAGAGCGTTGAGAATCTGCCAGGCGGCGGCGACTCTCGCTGGATTGGGATAGTTTTTGTTAGCCAGCATCACTA : } 80 \\ \text { D4 : } & \text { TTACTGTAGAGCGTTGAGAATCTGCCAGGCGGCGGCGACTCTCGCTGGATTGGGATAGTTTTTGTTAGCCAGCATCACGA }: 80\end{array}$

D107 : TACCCAGCTCTTTTTCTGGAATAAACGCGACATAGCTACCAAATCCGCCGGT TGCCCCTGTTTTATGTACCCATGATGCG : 160

D4 : TACCCAGCTCTTTTTCTGGAATAAACGCGACATAGCTACCAAATCCGCCGGT CGCCCCCGTTTTATGCACCCATGATGCG : 160

D107 : CGTACTGCAGGAGTTGGGGGCGTAATCGCTTTTACGGGGTGTGCTGCCAGTGCAATTTTATTGCCACTGCCGTTAATGAT : 240

D4 : CGTACTGCAGGAGTTGGGGGCGTAATCGCTTTTACGGGGCGTGCTGCCAGTGCAATTTTATTGTCACTGCCGTTAACGAT : 240

D107 : GCTGTCAGGATTTACCGGCCAGTCCAGCATTTCCCAGCCCAGGCCCTGATACATATCGCCGGTTTGCAGTAGCGAGATT : 320

D4 : GATGTCAGGATTTACCGGCCAGTCCAGCATTTCCCAGCCCAGACCCTGATACATATCGCCGGTTTGCCAGTAGCGAGATT : 320

D107 : GTGCCAGTTGTATCCCTTGCTGAAGTGTTTTGTCGTTGATATCACGGGGATTCATATTGCTTCGTACCCAGCAGGCCATA : 400

D4 : GTGCCAGTTGTATCCCTTGTTGAAGTGTTTTGTCGTTGATATCACGGTGATTCATATTGCTTCGTACCCAGCAGGCCATA : 400

D107 : TCTTCAATGGTCGACTTCACACCATAAGTTTCAGCATCTAACGCCCCTGGCGAAACATGAACTGCCTTGCCTTCGCGATA : 480

D4 : TCTTCAATGGTCGACTTCACACCATAAGTTTCAGCATCTAACGCCCCTGGCGAAACATGAACTGCCTTGCCTTCGCGATA : 480

D107 : TCCCCAGGCGTAATTCTTTTCTTCTGCGGGCGGTACATTAATCCACGTATGGTTGAGTTTGAGTGGCTGGAAGACACGTG : 560

D4 : TCCCCAGGCGTAATTCTTTTCTTCTGCGGGCGGTACATTAATCCACGTATGGTTGAGTTTGAGTGGCTGGAAGACACGTG : 560

D107 : TTTGCATCGCCTGCTCAAAACTCAAACCAGACGGCTTCACAGCCAGTGCGCCGAACAAACCGATACTGGAGTTGGCATAC : 640

D4 : TTTGCATCGCCTGCTCAAAACTCAAACCAGACGGCTTCACAGCCAGTGCGCCGAACAAACCGATACTGGAGTTGGCATAC : 640

D107 : AGACGTTGTGTTCCTGGCGCCCATGCAGGCTGCCAGTTTTGATAGAAGCGCAGCAAGTCGCTTGAGGATTTCACCTCATC : 720

D4 : AGACGTTGTGTTCCTGGCGCCCATGCAGGCTGCCAGTTTTGATAGAAGCGCAGCAAGTCGCTTGAGGATTTCACCTCATC : 720

D107 : CGGCACCTGCAATGGCAGGCGCCAGCAGTGTAGGTTGCGAGATGTAATAGTGTGATCCCATTCCACTGTTTAGCGGTAA : 800

D4 : CGGCACCTGCAATGGCAGGCCGCCAGTAGTGTAGGTTGCGAGATGTAATAGTGTGATCCCATTCCACTGTTTAGCGGTAA : 800

D107 : GTTCAGGCCAGTATTTTGTTGTGGGATCGCTTAACTTGATTTCCCCTCGAGCAATAGCGTCGCCACCAAGCACGCCCGTA : 880

D4 : GTTCAGGCCAGTATTTTGTTGTGGGATCGCTTAACTTGATTTCCCCTCGAGCAATAGCGTCGCCACCAAGCACGCCCGTA : 880

D107 : AATGTTTTGCTGACCGAACCTAACTCAAACAACGTTTGCTGTGTGACGGGCTGCTTTTTGGCGATGTCCGCATAGCCCCA : 960

D4 : AATGTTTTGCTGACCGAACCTAACTCAAACAACGTTTGCTGTGTGACGGGCTGCTTTTTGGCGATGTCCGCATAGCCCCA : 960

D107 : GGTAAAGTAATAAGGTTTACCCTGATAAATTACCGCCACCGCCATACCGGGGATCTTTTGTTGCTCTATAAGCGGGGTAA : 1040

D4 : GGTAAAGTAATAAGGTTTACCCTGATAAATTACCGCCACCGCCATACCGGGGATCTTTTGTTGCTCTATAAGCGGGGTAA : 1040

D107 : TTGTGCGATGCACAATATCGTTGATTTGTTGAGGGGCAGCAAATGTGGAGCAAGAGGCGGTAATTAATAAGGCGCAGAGC : 1120

D4 : TTGTGCGATGCACAATATCGTTGATTTGTTGAGGGGCAGCAAATGTGGAGCAAGAGGCGGTAATTAATAAGGCGCAGAGC : $: 1120$

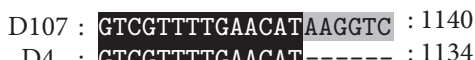

D4 : GTCGTTTTGAACAT------ : 1134

Figure 4: Alignment result of $A m p C$ gene in the genomes of China strain (D107) and Sudan strain (D4).

\section{References}

[1] S. Schjørring and K. A. Krogfelt, "Assessment of Bacterial Antibiotic Resistance Transfer in the Gut," International Journal of Microbiology, vol. 2011, Article ID 312956, 10 pages, 2011.

[2] D. L. Paterson, W.-C. Ko, A. Von Gottberg et al., "Antibiotic therapy for Klebsiella pneumoniae bacteremia: implications of production of extended-spectrum $\beta$-lactamases," Clinical Infectious Diseases, vol. 39, no. 1, pp. 31-37, 2004.

[3] C. A. Bunner, B. Norby, P. C. Bartlett, R. J. Erskine, F. P. Downes, and J. B. Kaneene, "Prevalence and pattern of antimicrobial susceptibility in Escherichia coli isolated from pigs reared under antimicrobial-free and conventional production methods," Journal of the American Veterinary Medical Association, vol. 231, no. 2, pp. 275-283, 2007. 
[4] G. M. Rossolini and M. C. Thaller, "Coping with antibiotic resistance: Contributions from genomics," Genome Medicine, vol. 2, no. 2, 2010.

[5] L. J. Piddock, "Does the use of antimicrobial agents in veterinary medicine and animal husbandry select antibiotic-resistant bacteria that infect man and compromise antimicrobial chemotherapy?" Journal of Antimicrobial Chemotherapy, vol. 38, no. 1, pp. 1-3, 1996.

[6] A. E. Van Den Bogaard and E. E. Stobberingh, "Antibiotic usage in animals. Impact on bacterial resistance and public health," Drugs, vol. 58, no. 4, pp. 589-607, 1999.

[7] C. T. Archer, J. F. Kim, H. Jeong et al., “The genome sequence of E. coli W (ATCC 9637): comparative genome analysis and an improved genome-scale reconstruction of E. coli," BMC Genomics, vol. 12, article 9, 2011.

[8] P. B. Eckburg, E. M. Bik, C. N. Bernstein et al., "Microbiology: diversity of the human intestinal microbial flora," Science, vol. 308, no. 5728, pp. 1635-1638, 2005.

[9] G. D. Wright, "The antibiotic resistome: the nexus of chemical and genetic diversity," Nature Reviews Microbiology, vol. 5, no. 3, pp. 175-186, 2007.

[10] N. Woodford and D. M. Livermore, "Infections caused by Gram-positive bacteria: a review of the global challenge," Infection, vol. 59, supplement 1, pp. S4-S16, 2009.

[11] G. J. Velicer, G. Raddatz, H. Keller et al., "Comprehensive mutation identification in an evolved bacterial cooperator and its cheating ancestor," Proceedings of the National Acadamy of Sciences of the United States of America, vol.103, no. 21, pp. 81078112, 2006.

[12] L. L. Marcusson, N. Frimodt-Møller, D. Hughes, and B. R. Levin, "Interplay in the Selection of Fluoroquinolone Resistance and Bacterial Fitness," PLoS Pathogens, vol. 5, no. 8, p. e1000541, 2009.

[13] L. Friedman, J. D. Alder, and J. A. Silverman, "Genetic changes that correlate with reduced susceptibility to daptomycin in Staphylococcus aureus," Antimicrobial Agents and Chemotherapy, vol. 50, no. 6, pp. 2137-2145, 2006.

[14] C. D. Herring, A. Raghunathan, C. Honisch et al., "Comparative genome sequencing of Escherichia coli allows observation of bacterial evolution on a laboratory timescale," Nature Genetics, vol. 38, no. 12, pp. 1406-1412, 2006.

[15] H. Hasman, D. Saputra, T. Sicheritz-Ponten et al., "Rapid whole-genome sequencing for detection and characterization of microorganisms directly from clinical samples," Journal of Clinical Microbiology, vol. 52, no. 1, pp. 139-146, 2014.

[16] S. Niemann, C. U. Köser, S. Gagneux et al., "Genomic diversity among drug sensitive and multidrug resistant isolates of Mycobacterium tuberculosis with identical DNA fingerprints," PLoS ONE, vol. 4, no. 10, Article ID e7407, 2009.

[17] A. E. van den Bogaard, N. London, C. Driessen, and E. E. Stobberingh, "Antibiotic resistance of faecal Escherichia coli in poultry, poultry farmers and poultry slaughterers," Journal of Antimicrobial Chemotherapy, vol. 47, no. 6, pp. 763-771, 2001.

[18] O. O. Adelowo, F. A. Ojo, and O. E. Fagade, "Prevalence of multiple antibiotic resistance among bacterial isolates from selected poultry waste dumps in Southwestern Nigeria," World Journal of Microbiology and Biotechnology, vol. 25, no. 4, pp. 713-719, 2009.

[19] P. M. Hawkey and A. M. Jones, "The changing epidemiology of resistance," Journal of Antimicrobial Chemotherapy, vol. 64, supplement 1, pp. i3-i10, 2009.
[20] T. D. Miles, W. McLaughlin, and P. D. Brown, "Antimicrobial resistance of Escherichia coli isolates from broiler chickens and humans," BMC Veterinary Research, vol. 2, article 7, pp. 1-9, 2006.

[21] M. Marchant, L. Vinué, C. Torres, and M. A. Moreno, "Change of integrons over time in Escherichia coli isolates recovered from healthy pigs and chickens," Veterinary Microbiology, vol. 163, no. 1-2, pp. 124-132, 2013.

[22] M. Čižman, "The use and resistance to antibiotics in the community," International Journal of Antimicrobial Agents, vol. 21, no. 4, pp. 297-307, 2003.

[23] V. I. Enne, C. Cassar, K. Sprigings, M. J. Woodward, and P. M. Bennett, "A high prevalence of antimicrobial resistant Escherichia coli isolated from pigs and a low prevalence of antimicrobial resistant E. coli from cattle and sheep in Great Britain at slaughter," FEMS Microbiology Letters, vol. 278, no. 2, pp. 193-199, 2008.

[24] B. Guerra, E. Junker, A. Schroeter, B. Malorny, S. Lehmann, and R. Helmuth, "Phenotypic and genotypic characterization of antimicrobial resistance in German Escherichia coli isolates from cattle, swine and poultry," Journal of Antimicrobial Chemotherapy, vol. 52, no. 3, pp. 489-492, 2003.

[25] F. Martineau, F. J. Picard, N. Lansac et al., "Correlation between the resistance genotype determined by multiplex PCR assays and the antibiotic susceptibility patterns of Staphylococcus aureus and Staphylococcus epidermidis," Antimicrobial Agents and Chemotherapy, vol. 44, no. 2, pp. 231-238, 2000.

[26] L. P. Randall, S. W. Cooles, M. K. Osborn, L. J. V. Piddock, and M. J. Woodward, "Antibiotic resistance genes, integrons and multiple antibiotic resistance in thirty-five serotypes of Salmonella enterica isolated from humans and animals in the UK," Journal of Antimicrobial Chemotherapy, vol. 53, no. 2, pp. 208-216, 2004. 


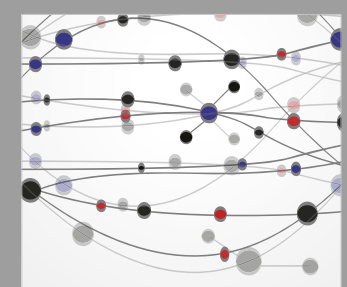

The Scientific World Journal
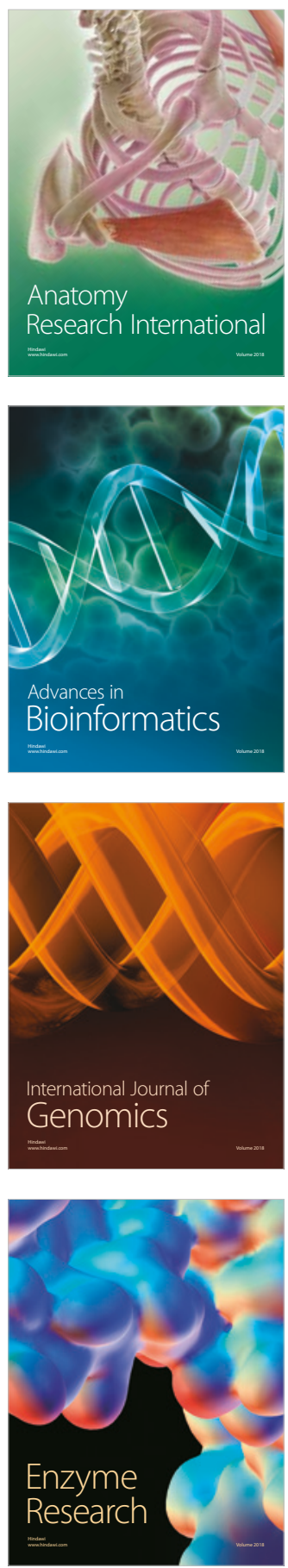
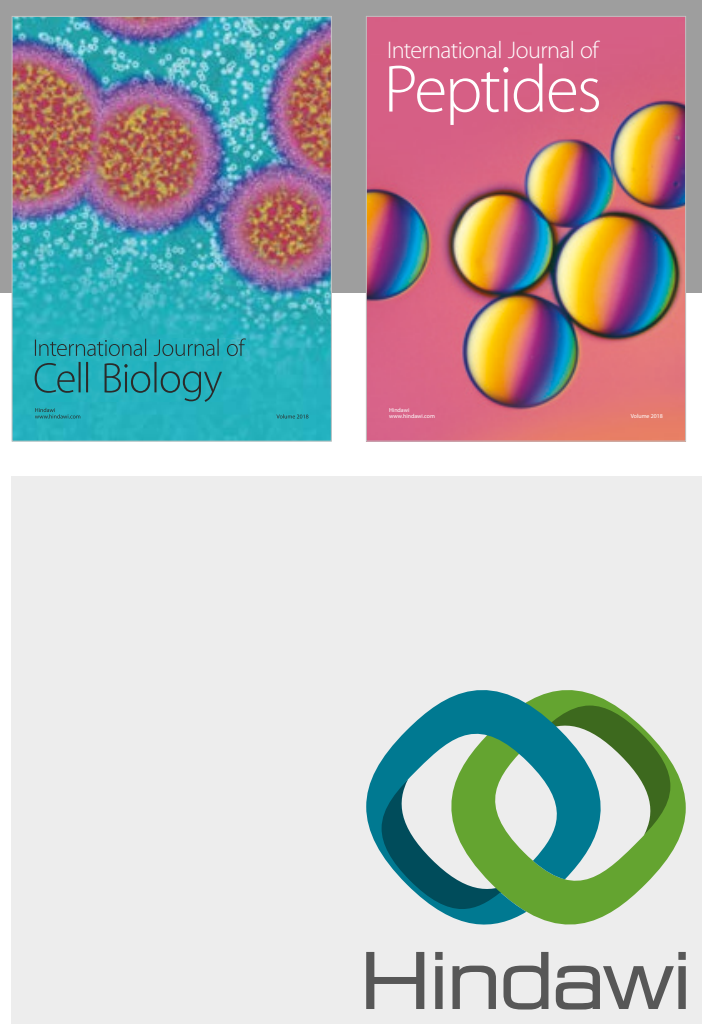

Submit your manuscripts at

www.hindawi.com
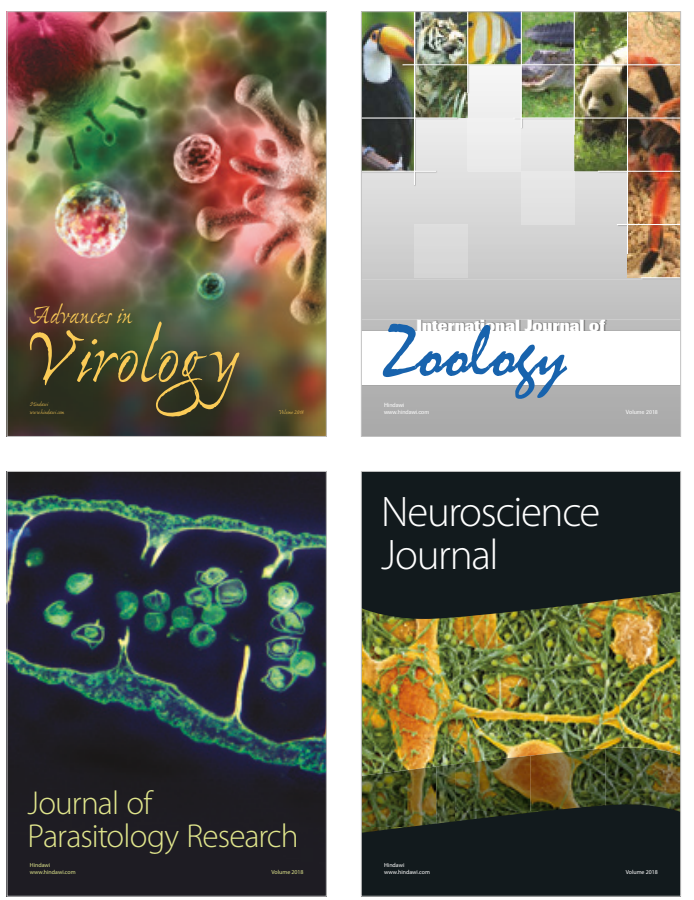
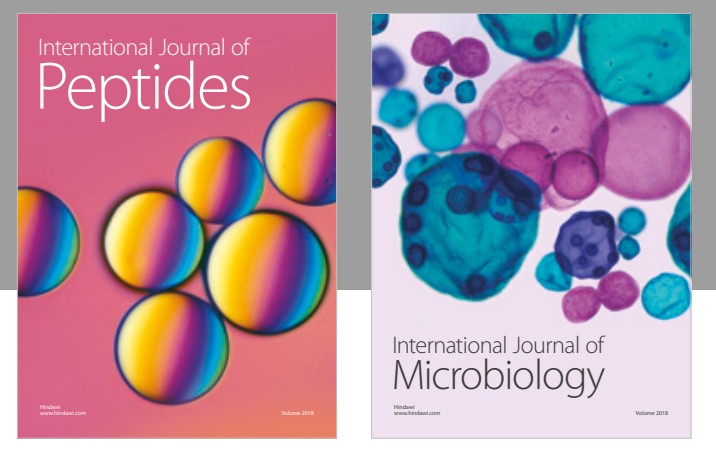

nternational Journal of Microbiology
Journal of
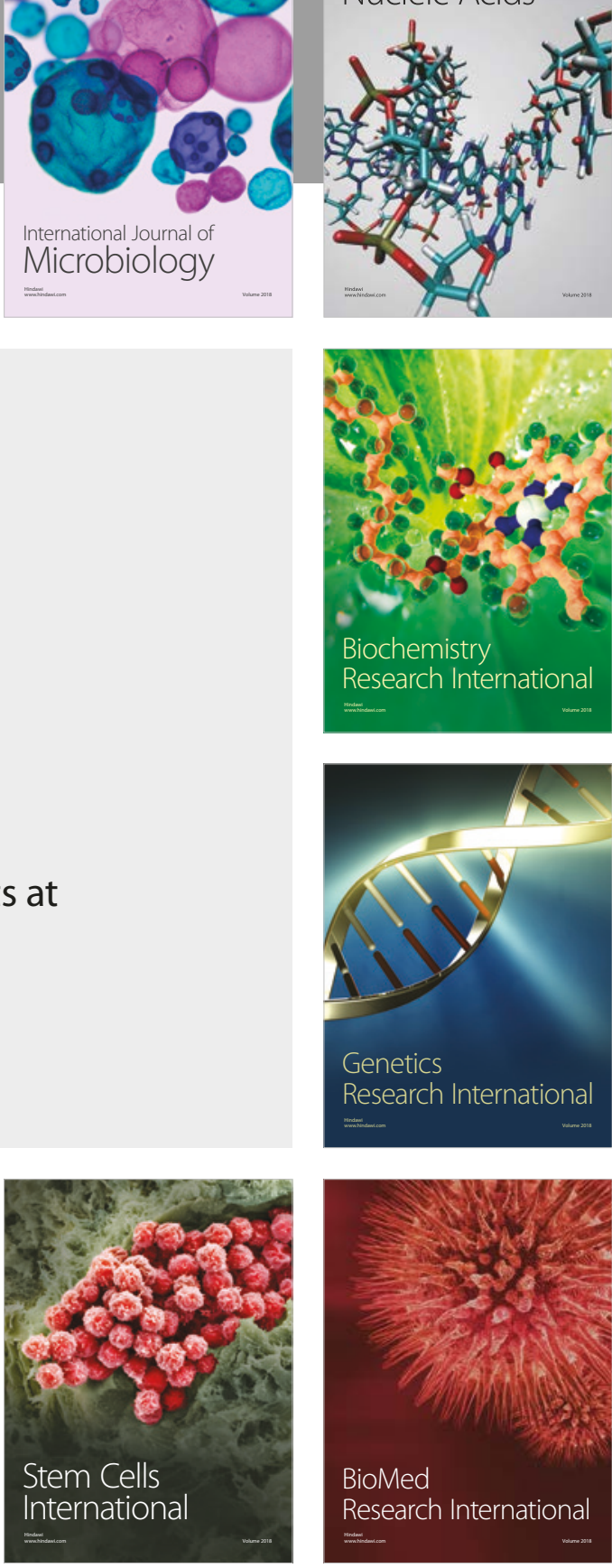
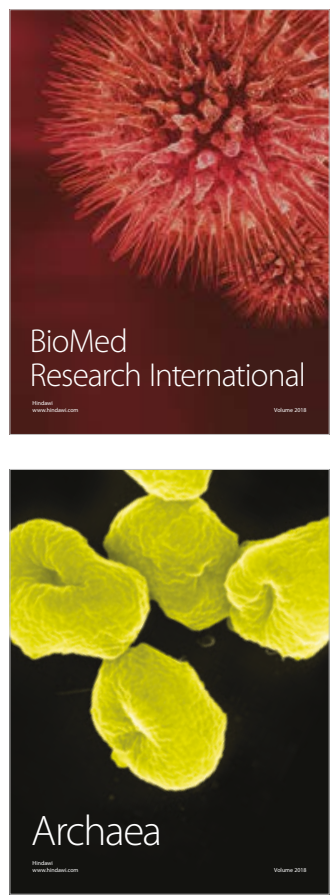